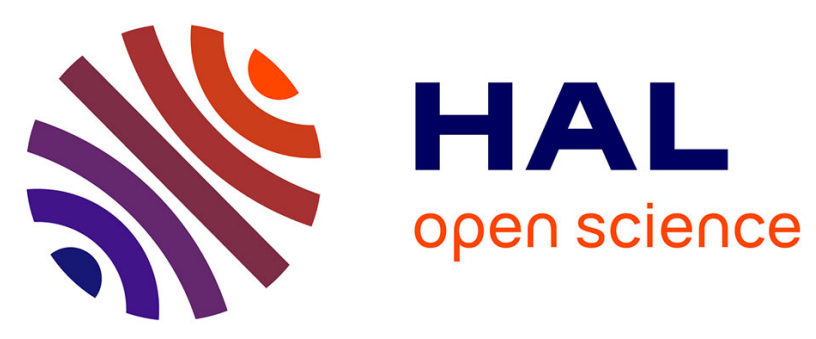

\title{
Combination of guided mode and photometric optical metrology methods for precise determination of refractive index dispersion: application to polymer blend and ceramic thin films for gas sensors
}

Thomas Wood, Judikaël Le Rouzo, François Flory, Paul Coudray, Valmor Roberto Mastelaro, Pedro Pelissari, Sergio Zilio

\section{To cite this version:}

Thomas Wood, Judikaël Le Rouzo, François Flory, Paul Coudray, Valmor Roberto Mastelaro, et al.. Combination of guided mode and photometric optical metrology methods for precise determination of refractive index dispersion: application to polymer blend and ceramic thin films for gas sensors. Optical Engineering, 2013, 52 (9), 10.1117/1.OE.52.9.094104 . hal-01810803

\section{HAL Id: hal-01810803 https://hal.science/hal-01810803}

Submitted on 15 Nov 2021

HAL is a multi-disciplinary open access archive for the deposit and dissemination of scientific research documents, whether they are published or not. The documents may come from teaching and research institutions in France or abroad, or from public or private research centers.
L'archive ouverte pluridisciplinaire HAL, est destinée au dépôt et à la diffusion de documents scientifiques de niveau recherche, publiés ou non, émanant des établissements d'enseignement et de recherche français ou étrangers, des laboratoires publics ou privés. 


\section{Optical Engineering}

Combination of guided mode and photometric optical metrology methods for precise determination of refractive index dispersion: application to polymer blend and ceramic thin films for gas sensors

Thomas Wood

Judikaël Le Rouzo

François Flory

Paul Coudray

Valmor Roberto Mastelaro

Pedro Pelissari

Sergio Zilio 


\section{Combination of guided mode and photometric optical metrology methods for precise determination of refractive index dispersion: application to polymer blend and ceramic thin films for gas sensors}

\author{
Thomas Wood \\ Judikaël Le Rouzo \\ Aix-Marseille University \\ Institut Matériaux Microélectronique Nanosciences \\ de Provence-IM2NP \\ CNRS-UMR 6242, Domaine Universitaire de \\ Saint-Jérôme \\ Service 231, 13397 Marseille, France \\ E-mail: tomwood321@ @otmail.com \\ François Flory \\ Aix-Marseille University \\ Institut Matériaux Microélectronique Nanosciences \\ de Provence-IM2NP \\ CNRS-UMR 6242, Domaine Universitaire de \\ Saint-Jérôme \\ Service 231, 13397 Marseille, France \\ and \\ Ecole Centrale Marseille \\ Technopôle de Château Gombert \\ Marseille 13013, France

\section{Paul Coudray} \\ Kloé SA \\ Hotel d'Entreprise du Millenaire \\ Montpellier 34000, France \\ Valmor Roberto Mastelaro \\ Pedro Pelissari \\ Sergio Zilio \\ Sao Paulo University \\ Instituto de Física de São Carlos \\ São Carlos 13560, Brazil
}

\begin{abstract}
Two optical techniques, m-lines and spectroscopic ellipsometry, are compared for their suitability for obtaining the wavelength and the temperature dispersion of the refractive index of thin-film layers used in gas detector devices. Two types of materials that are often integrated into gas sensors are studied: a polymer organic-inorganic blend deposited by spin coating typically used in near-infrared waveguides and the ceramic semiconductor $\mathrm{SrTi}_{1-x} \mathrm{Fe}_{x} \mathrm{O}_{3}$ (strontium titanate) doped with iron at concentrations $x=0.075$ and 0.1 deposited by electron beam deposition. The refractive index dispersion obtained by $m$-lines and ellipsometry is compared, and the differences between the measured parameters for the two materials are discussed. The chromatic dispersion will be represented by a three-term Cauchy law. An intuitive method for verifying the measured indices using an integrating sphere and reflexion coefficient modeling techniques are also demonstrated. Thermo-optic coefficients in the order of $-1 \times 10^{-4} / \mathrm{K}$ for both materials are reported, and very low chromatic dispersions are also measured, thanks to the high sensitivity of the $\mathrm{m}$-lines technique. The uniaxial anisotropic properties of the polymerblend films are measured and discussed in the case of the semiconductor films. ( 2013 Society of Photo-Optical Instrumentation Engineers (SPIE) [DOI: 10.1117/1.OE .52.9.094104]
\end{abstract}

Subject terms: refractive index dispersion; thermo-optic coefficient; uniaxial anisotropy; ellipsometry; m-lines; thin films; ceramic; polymer blend.

Paper 130529P received Apr. 4, 2013; revised manuscript received Jul. 10, 2013; accepted for publication Aug. 16, 2013; published online Sep. 10, 2013.

\section{Introduction}

For several decades, the use of dielectric materials as gas sensors has been limited to measure changes in their electrical properties, for example conductivity, in response to the target gas. ${ }^{1}$ More recently, attention has been turned to variations in the optical properties of dielectric thin films, namely the complex refractive index. ${ }^{2}$ The advantages of optical over electrical detection are many, ranging from a reduction in energy consumption of the detector, due to improved performances at lower temperatures, to an improved sensitivity inherent to optical interferometric devices. In order to incorporate a material into an optical transduction system, it is important to have a full knowledge of how the said material's properties vary as a function of wavelength and temperature. This is essential as it allows the designer of the detector to specify at a later stage the probe wavelength that the device

0091-3286/2013/\$25.00 @ 2013 SPIE will employ, as well as to perform simulations of the performances of a given optical architecture so as to tune the detector. From a thermal point of view, the dispersion of the refractive index as a function of temperature allows one to distinguish between changes in the detector output, due to ambient temperature variations, and a true response to the target gas.

An emerging technology in the gas sensor market employs catalytic oxidation of certain chemical species on the surface of an optical transducer, and the heat released by such exothermic reactions being captured, so as to modify the optical properties of the materials used. For such systems, the key parameter to be determined for materials interacting with light is the thermo-optic coefficient, which is defined as the first derivative of the real part of the refractive index with respect to the temperature. The quantity typically takes an absolute value of the order $10^{-4} / \mathrm{K}$, a negative value indicating a decrease of the refractive index with an increase in temperature. It is therefore necessary to use a measurement 
system with a very high sensitivity, in order to detect the change in refractive index for small temperature variations. The behavior of the optical transducer is often polarization-dependent, and as such precise measurements of the birefringence of the constituent materials are necessary, so as to predict the device's optical output.

\section{Sample Preparation}

\subsection{Polymer-Blend Samples}

The polymer-blend materials characterized in this article are hybrid organic-inorganic mixtures deposited by spin coating, which is developed by the KLOE Company in Montpellier, France. They are designed to have low losses-hence, a low extinction coefficient - in the visible to near-infrared spectral range, facilitating their use in waveguide fabrication. Two different materials are studied: the first being referred to as a buffer material and the second as a guiding material, due to their roles when used to constitute a waveguide. The guiding material — of a slightly higher refractive index - in which light is confined is actually obtained from the buffer material by exposing the latter to UV radiation. This technology constitutes KLOE's patented Dilase system. For our purposes, several sample sets were prepared: the first being a two-layer stack on a silicon wafer substrate consisting of a $14-\mu \mathrm{m}$ buffer layer on the substrate topped with a $4-\mu \mathrm{m}$ guiding layer. These samples were used for ellipsometric chromatic dispersion measurements for both materials as well as for thermal and chromatic dispersion measurements of the guiding film with the m-lines setup. The second sample set consisted of single thin films of approximately $5-\mu \mathrm{m}$ thickness of each material deposited separately on AF32eco glass substrates. These samples were used in order to obtain the chromatic dispersion and to measure the anisotropic properties of the films by m-lines, as well as to provide information used in the ellipsometry data treatment stage. It should be noted that due to the semiorganic nature of the polymer blend materials, their temperature should not exceed $80^{\circ} \mathrm{C}$. Above this temperature, irreversible damage may be caused, and the film may peel off from the substrate.

\section{$2.2 \mathrm{SrTi}_{1-x} \mathrm{Fe}_{x} \mathrm{O}_{3}$ Ceramic Samples}

The doped strontium titanate ceramic samples $\left(\mathrm{SrTi}_{1-x} \mathrm{Fe}_{x} \mathrm{O}_{3}\right.$, referred to as "STF") were prepared in Brazil at the Instituto de Fisica de Sao Carlos (University of Sao Paulo). ${ }^{3,4}$ Doping levels of $7.5 \%$ and $10 \%$ iron, corresponding to $x=0.075$ and 0.1 , were selected based on previous studies that showed that these concentrations gave the most promising electrical results in response to oxidizing gases such as ozone. First, citric acid (CA) and iron nitrate were dissolved in distilled water at room temperature under constant agitation. After complete dissolution, the temperature of the solution was raised to $80^{\circ} \mathrm{C}$, and the $\mathrm{SrCO}_{3}$ was slowly added to the aqueous solution until it became transparent. Then, ethylene glycol (EG) was added into this solution, and it was heated up to $150^{\circ} \mathrm{C}$. The molar ratio among the cations $(\mathrm{Sr}+\mathrm{Fe})$ and the CA was $4: 1$. Subsequently, the solution of titanium citrate was prepared in another beaker. The citrate was formed by dissolution of $\mathrm{CA}$ and titanium isopropoxide in distilled water at $70^{\circ} \mathrm{C}$, and the $\mathrm{CA}$ : Ti molar ratio was $4: 1$. After complete homogenization of the citrate solution, the EG was added into the solution at a mass ratio of $40: 60$, in relation to $\mathrm{CA}$. The $\mathrm{Sr}-\mathrm{Fe}-\mathrm{Ti}$ resin was prepared by mixing strontium-iron and titanium solutions kept at molar ratio $1: 1$ $(\mathrm{Sr}: \mathrm{Ti}+\mathrm{Fe})$, obtaining a colorless resin. Then, the temperature of solution was raised at $150^{\circ} \mathrm{C}$, for the elimination of water, until the solution acquired a viscous aspect, and then heat treated at $300^{\circ} \mathrm{C}$ for $8 \mathrm{~h}$ with a heating rate of $10^{\circ} \mathrm{C} \mathrm{min}{ }^{-1}$, in order to eliminate the organic species. These powders were called "precursors." These precursor powders were calcined at $700^{\circ} \mathrm{C}$ for $1 \mathrm{~h}$ (two times) in an electric furnace under air atmosphere. X-Ray diffraction (XRD) measurements are performed at this stage to confirm the crystalline nature of the product. The resulting powder is pressed into disc-shaped pellets (diameter $1 \mathrm{~cm}$ and depth approximately $0.5 \mathrm{~cm}$ ). STF thin films were deposited in a Balzers BAK600 evaporator using the prepared pellets as a target. Thicknesses of 190 to $250 \mathrm{~nm}$ were obtained at a rate of $10 \mathrm{~nm} / \mathrm{s}$, while keeping the substrates at a temperature of $50^{\circ} \mathrm{C}$. The substrates were attached to a holder rotating at $23 \mathrm{rpm}$ in the plane perpendicular to the incident flux of deposited material at a distance of $60 \mathrm{~cm}$ from the crucible containing the pellet. The atmosphere in the deposition chamber was that of pure oxygen atmosphere at a pressure of $2 \times 10^{-4}$ mbar. During evaporation, the thicknesses were monitored with a quartz balance, and at the end of the process, we confirmed the thicknesses by means of a profilemeter. The evaporation was assisted by an electron beam operating at $6 \mathrm{kV}$ and $0.2 \mathrm{~A}$ accelerating electrons from a filament supplied with a 40-A current.

\section{Measurement Techniques}

\section{1 m-Lines Measurements}

The "m-lines" technique provides excellent experimental precision, due to the increased light-material interaction inherent to guided mode techniques, but only at a discrete set of wavelengths corresponding to the output of available lasers. While satisfactory for materials presenting an easily modeled dispersion over the spectral region of interest, such as the studied polymer-blend film, the limits of this technique are evident when materials with more complex index dispersions are studied. In order to extract sufficient information to establish the true chromatic dispersion for such materials, for example the ceramic films presented in this article, a more broadband technique such as spectroscopic ellipsometry must be employed.

\subsubsection{Isotropic thin films}

The principle of m-lines is the excitation of guided modes in thin films through the use of a prism coupler in close proximity to the said film. ${ }^{5}$ For incident angles of light on the prism base for which the propagation vector matches that of a guided mode, optical energy is transferred from the prism to the film. The setup consists of a Gaussian laser beam focused onto the prism base in a total reflection setup, and thereby providing an angular range of incident angles. By rotating the prism coupler, one can change the range of incident angles. When the matching condition of the propagation vectors is satisfied, a dark line-called an $\mathrm{m}$-line-appears in the totally reflected beam, indicating that the optical energy has been transferred to the film. When the prism-layer coupling is uniform and the layer 


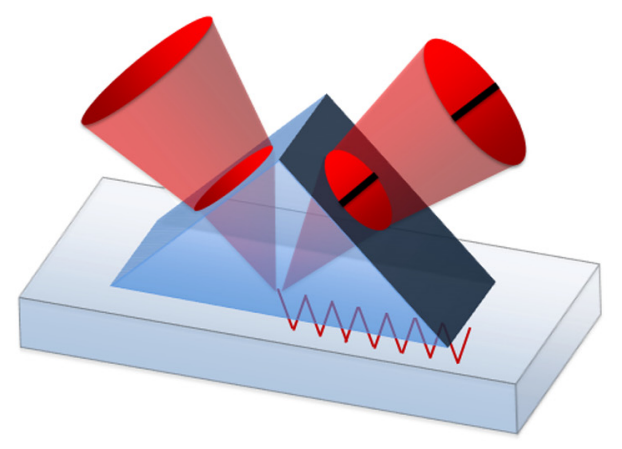

(a)

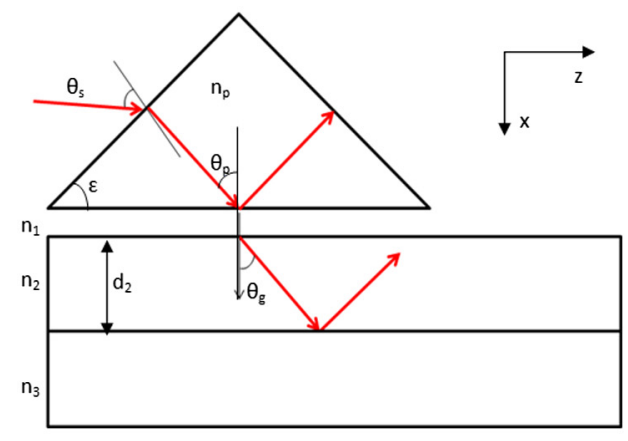

(b)

Fig. 1 (a) Three-dimensional representation of the m-lines prism coupler system and (b) schematic representation showing the geometrical parameters linked to find the propagation constant.

has low losses, the dark line may be followed by a bright line and further interference fringes. This phenomenon can be fully modeled for an incident Gaussian beam with rigorous electromagnetic theory. ${ }^{6}$ Measuring the corresponding incident angles for two such m-lines, known as synchronous angles, coupled with knowledge of the prism parameters (refractive index and characteristic angle) and the refractive index of the substrate, one can numerically resolve a transcendental equation to obtain both the refractive index and the thickness of the guiding film. This procedure can be performed in the two polarizations: transverse electric (TE), in which the electric field is entirely contained in the plane of the substrate, and transverse magnetic (TM), in which the magnetic field lies in the substrate plane. We may also note that if either the refractive index or the thickness of the film is known, the remaining parameter may be calculated from the angular position of a single m-line.

The chromatic dispersion of the index of thin films can be determined by using a laser with multiple wavelength outputs such as the HeNe laser. ${ }^{7}$ The temperature dispersion is obtained with the use of a variable hot air flow and a surface thermocouple in contact with the guiding film to measure its temperature. ${ }^{8}$ In these two cases, care must be taken to ensure that the variation of the refractive index of the prism as a function of wavelength and temperature are integrated into the calculations (Fig. 1).

The number of guided modes supported by a thin film per polarization depends on its optical thickness. For the ceramic films characterized, only one mode could be supported per polarization. Due to the fact that the setup is quite restrictive in terms of angular displacement of the prism coupler once the heating and temperature detection systems have been installed, it was not possible to measure the angular position of two m-lines per studied temperature. This is due to the fact that the synchronous angles for the TE polarization are significantly different for those in TM because of the high birefringence of the used rutile $\left(\mathrm{TiO}_{2}\right)$ prism. In this case, it is still possible to calculate the refractive index of the film from one synchronous angle by supposing a constant thickness calculated before the installation of the heating equipment. The measurement uncertainty on absolute values of the refractive index is typically in the order of $10^{-3}$ for goodquality films. ${ }^{9}$ The technique is, however, sensitive to variations in the index in the order of $10^{-6}$; this being roughly 100 times inferior to the change in index observed for a $1 \mathrm{~K}$ change in film temperature.

\subsubsection{Anisotropic thin films (uniaxial)}

Materials deposited in thin films can present very different optical properties than those observed in their bulk counterparts. One of the most common particularities is the presence of an optical anisotropy, most often uniaxial, which exposes the components of the electric field in the plane of the film/ substrate interface to a different refractive index to the components directed following the thickness of the film. The origin of this phenomenon lies in the employed deposition technique. For thin films deposited by sol-gel methods, such as the polymer-blend materials studied here, the material constituting the coating is dissolved in a solvent and spread on the substrate by mechanical means such as spin coating or dip coating. When the solvent evaporates, the quantity of material composing the film decreases, leading to a shrinking in the direction following the thickness of the film (perpendicular to the film/substrate interface). On the other hand, the adherence between the deposited material and the substrate prevents the film from contracting in the interface plane. The mechanical constraints induced lead to a uniaxial anisotropy of the refractive index of the film, ${ }^{10}$ as illustrated in Fig. 2.

For a guided mode in TM polarization in a thin film, the electric field $E_{\mathrm{TM}}$ can be decomposed into two components: $E_{\mathrm{TM}, y}$ (following the $y$-axis), which experiences refractive index $n_{o}$, and $E_{\mathrm{TM}, z}$ (following the $z$-axis), which experiences the refractive index $n_{e}$. The amplitude of each component depends on the propagation angle of the light in the film, and hence, varies for each guided mode. One can write the following equation linking the electric field components and the experienced refractive indices:

$\frac{\left|E_{\mathrm{TM}}\right|^{2}}{n_{\theta_{g}}^{2}}=\frac{\left|E_{\mathrm{TM}, y}\right|^{2}}{n_{o}^{2}}+\frac{\left|E_{\mathrm{TM}, x}\right|^{2}}{n_{e}^{2}}$,

where $n_{\theta}$ is the refractive index experienced by a guided mode with propagation angle $\theta_{g}$.

This can be rewritten as

$$
\frac{1}{n_{\theta_{g}}^{2}}=\frac{\cos ^{2} \theta_{g}}{n_{o}^{2}}+\frac{\sin ^{2} \theta_{g}}{n_{e}^{2}} .
$$

The variation of the refractive index with the propagation angle for light exhibiting TM polarization in uniaxially anisotropic films allows for the calculation of the two refractive 


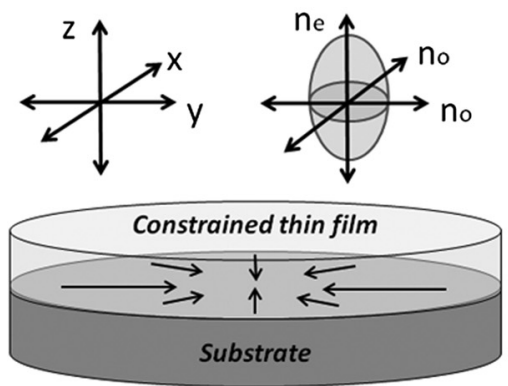

(a)

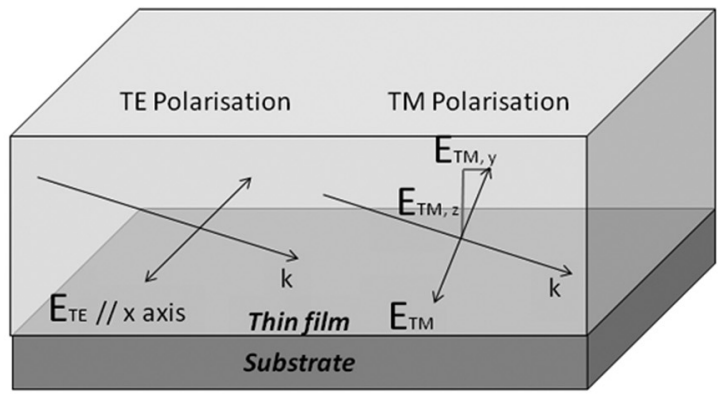

(b)

Fig. 2 (a) Diagram showing schematically the nature of the constraints in sol-gel thin films as well as the coordinate system and the refractive index. $\langle x y\rangle=$ film/substrate interface plan, refractive index $n_{o}, z=$ direction defining film thickness, refractive index $n_{e}$. (b) Decomposition of TM electric field using Cartesian coordinate system.

indices present in the film through the combination of measurements made in TE and TM polarizations. First, the angular position of two m-lines is measured in TE polarization, which allows for the calculation of the ordinary index and the thickness of the film, as described earlier. Next, the angular position of one m-line is measured in TM polarization, which allows for the calculation of the effective index of this guided mode and the angular-dependent refractive index experienced by the guided mode electric field (using the film thickness from the TE measurements). By rearranging Eq. (2), one obtains the following expression for the calculation of the extraordinary refractive index from the ordinary index, the angular-dependent index, and the effective index of the TM mode:

$n_{e}=\sqrt{\frac{n_{o}^{2} N_{\mathrm{TM}}^{2}}{n_{o}^{2}-n_{\theta_{g}}^{2}+N_{\mathrm{TM}}^{2}}}$,

where $N_{\mathrm{TM}}=n_{\theta_{g}} \sin \theta_{g}$ is the effective index of a TMguided mode.

We should note that this method requires the presence of two or more guided modes in the TE polarization. As such, the ceramic films could not be measured using this technique, as their low optical thickness permits only the excitation of one guided mode per polarization. A discussion will be provided later on the presence of a uniaxial anisotropy in these films. The polymer blend films present sufficient optical thicknesses to support multiple guided modes per polarization. As such, their ordinary and extraordinary refractive indices have been measured using the method detailed earlier.

\subsection{Spectroscopic Ellipsometry Measurements}

Spectroscopic ellipsometry is a well-established technique for measuring the complex refractive index and the thickness of thin film stacks. It provides more complete information on the chromatic dispersion of a sample's refractive index, due to the fact that the broadband source allows the user access to a continuous probe spectrum. It should, however, be noted that the uncertainty on the measured refractive index is similar to that common to all photometric techniques, being in the order of $10^{-2}$. The temperature dispersion of the refractive index was also measured through the use of a heating stage placed under the sample during measurements. It should be noted that ellipsometry was not used to establish the temperature dispersion of the polymer-blend samples, the thermo-optic coefficient being in the order of $-1 \times 10^{-4} / \mathrm{K}$; in order to induce a change in the refractive index measurable with any degree of precision by ellipsometry, one would have to heat the sample to a temperature in the order of $100^{\circ} \mathrm{C}$. As previously stated, this would cause irreversible damage to the polymer-blend material.

The ellipsometry measurements presented were obtained using a SOPRA GES-5 spectroscopic ellipsometer equipped with a halogen source. The ellipsometer was configured to work in rotating polarizer mode with the analyzer pass angle fixed at $30 \mathrm{deg}$. An incidence angle of $65 \mathrm{deg}$ was chosen to be close to the Brewster angle of the substrate, so as to give the greatest possible variations in the $\cos \Delta$ (phase difference) term of the measured ellipsometric parameter $\rho$. In the data treatment stage, the real-part refractive index of the transparent polymer blend films was modeled using a simple Cauchy model. For the ceramic materials, a Cauchy law was used for both the real and imaginary parts of the refractive index along with an absorption peak displaying a Lorentzien distribution. ${ }^{11}$

\subsection{Integrating Sphere Measurements}

The measurements obtained from integrating sphere measurements are those of the global reflection and transmission coefficients ( $R_{\text {measured }}$ and $\left.T_{\text {measured }}\right)$ of the film-substrate systems. For our integrating sphere, the incident angle for light onto the sample is $8 \mathrm{deg}$ in both transmission and reflection configurations. The measurement of such photometric coefficients over a range of wavelengths corresponding to that chosen for ellipsometric measurements allows one to check the quality of the results obtained from ellipsometry via the construction of simulated reflection and transmission coefficients from the refractive indices and film thicknesses. ${ }^{12,13}$ The interaction of light with a thin-film stack can be studied using the Abeles formalism, ${ }^{14}$ wherein each film $j$ in a stack of $m$ layers can be represented with a complex propagation matrix.

$$
\begin{gathered}
\hat{C}_{j_{p, s}}=\left(\begin{array}{cc}
e^{-i \delta_{j-1}} & \hat{r}_{j p, s} e^{-i \delta_{j-1}} \\
\hat{r}_{j p, s} e^{i \delta_{j-1}} & e^{i \delta_{j-1}}
\end{array}\right) \\
\text { where } \delta_{j-1}=k_{0} \cdot \hat{N}_{j-1} \cos \theta_{j-1} d_{j-1},
\end{gathered}
$$

where $j$ is the numbering index of the films, $k_{0}=2 \pi / \lambda_{0}$ is the wavenumber of the incident light, $N_{j}$ is the complex refractive index of a film, $\theta_{j}$ is the propagation angle in a 
film, $d_{j}$ is the thickness of a film, and $r_{j}$ is the Fresnel reflection coefficient between the films $j-1$ and $j$.

The electric field is decomposed into two components at each interface: $E_{j}^{+}$and $E_{j}^{-}$, incident and reflected, respectively. One can relate the field at and interface $j$ with that incident on the stack $E_{0}$, thus

$$
\left(\begin{array}{c}
E_{0}^{+} \\
E_{0}^{-}
\end{array}\right)=\frac{\hat{C}_{1} \hat{C}_{2} \ldots \hat{C}_{j}}{\hat{t}_{1} \hat{t}_{2} \ldots \hat{t}_{j}}\left(\begin{array}{c}
E_{j}^{+} \\
E_{j}^{-}
\end{array}\right),
$$

where $C_{j}$ is the propagation matrix for the film $j$ [Eq. (5)] and $t_{j}$ is the Fresnel transmission coefficient at the interface $j$.

With this mathematical system, the global-simulated reflection and transmission coefficients for a stack of $m$ layers in $p$ (TM) and $s$ (TE) polarizations may be calculated as follows:

$$
\begin{aligned}
& R_{\text {simulated }(p, s)}=\left|\frac{\hat{c}_{p, s}}{\hat{a}_{p, s}}\right|^{2} \\
& T_{\text {simulated }(p)}=\operatorname{Re}\left(\frac{\hat{N}_{m+1}^{*} \cos \theta_{m+1}}{\hat{N}_{0}^{*} \cos \theta_{0}}\right)\left|\frac{\hat{t}_{1_{p}} \hat{t}_{2_{p}} \ldots \hat{t}_{j_{p}}}{\hat{a}_{p}}\right|^{2} \\
& T_{\text {simulated }(s)}=\operatorname{Re}\left(\frac{\hat{N}_{m+1} \cos \theta_{m+1}}{\hat{N}_{0} \cos \theta_{0}}\right)\left|\frac{\hat{t}_{1_{s}} \hat{t}_{2_{s}} \ldots \hat{t}_{j_{s p}}}{\hat{a}_{s}}\right|^{2},
\end{aligned}
$$

where $c$ and $a$ are elements of the matrix describing the stack, which is defined as follows:

$$
\hat{C}_{1 p, s} \hat{C}_{2 p, s} \ldots \hat{C}_{m_{p, s}}=\left(\begin{array}{ll}
\hat{a}_{p, s} & \hat{b}_{p, s} \\
\hat{c}_{p, s} & \hat{d}_{p, s}
\end{array}\right) .
$$

The comparison of these simulated spectra with those measured by the integrating sphere allows us to comment on the accuracy of the refractive indices and the thicknesses calculated from ellipsometry. It should be noted that due to the high-optical thickness of the polymer blend samples deposited on silicon ( $4 \mu \mathrm{m}$ for the guiding layer), the measured reflectance coefficient is dominated by thin-film coherent interference effects. As such, these measurements are not used to verify the ellipsometric measurements for such samples. It has also been observed for thicker films (over $10-\mu \mathrm{m}$ thickness) that such interference effects are no longer present, as the coherence length of the source is exceeded. For the ceramic samples that present a highextinction coefficient in the visible domain, the absorbance of the sample may be calculated from measurements of $R$ and $T$ using the assertion that for values of the photometric coefficients normalized to percentages $R+T+A=100$, the source intensity is conserved. In the visible domain, the absorption in the B270 substrate is negligible, and so all absorption can be attributed to the ceramic films. The absorbance as a function of wavelength is used to verify the extinction coefficient obtained by ellipsometry for the ceramic samples through the calculation of the theoretical absorbance: $A_{\text {simulated }}=100-R_{\text {simulated }}-T_{\text {simulated }}$.

\section{Results}

The chromatic and thermal dispersions of materials are represented, where possible, by the nine coefficients of a modified Cauchy law: ${ }^{15}$

$$
\begin{aligned}
n(\lambda, T)=A(T)+\frac{B(T)}{\lambda^{2}} \\
+\frac{C(T)}{\lambda^{4}}\left\{\begin{array}{l}
A(T)=a_{1}+a_{2} T+a_{3} T^{2} \\
B(T)=b_{1}+b_{2} T+b_{3} T^{2} \\
C(T)=c_{1}+c_{2} T+c_{3} T^{2}
\end{array}\right.
\end{aligned}
$$

This representation allows for the calculation of the refractive index at intermediate wavelengths and temperatures as well as for the extrapolation in a limited domain around the measured ranges. The calculation of these nine coefficients necessitates the measurement of the refractive index at three wavelengths at each of three different temperatures. In addition to the Cauchy parameters, the thermooptic coefficient is also given.

\subsection{Polymer Blend Films}

The chromatic dispersion of the refractive index at room temperature $\left(24^{\circ} \mathrm{C}\right)$, as calculated by m-lines and ellipsometry, is given in Fig. 3. One can clearly observe a linear shift

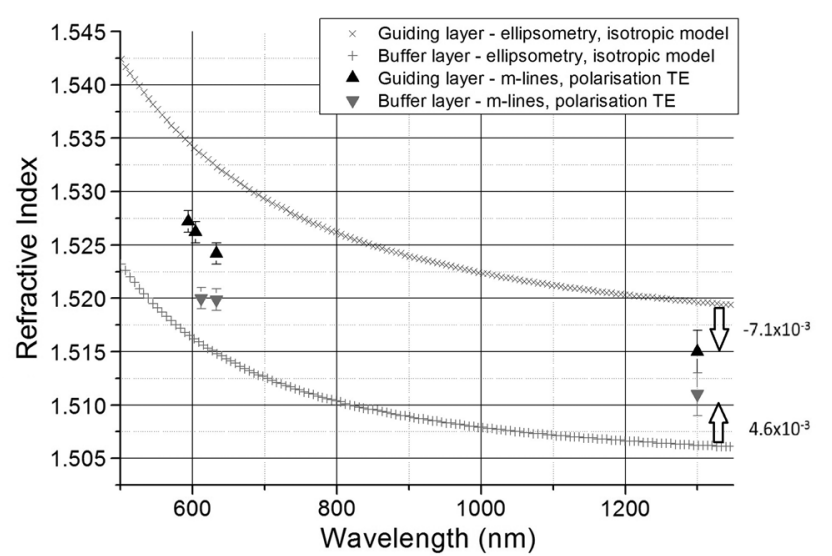

(a)

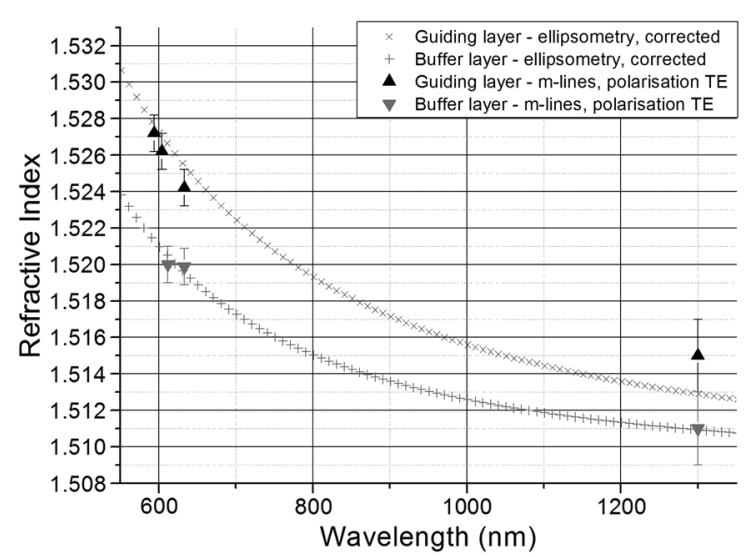

(b)

Fig. 3 (a) Chromatic dispersion obtained by m-lines and ellipsometry and (b) same after application of linear correction to ellipsometry measurements. 


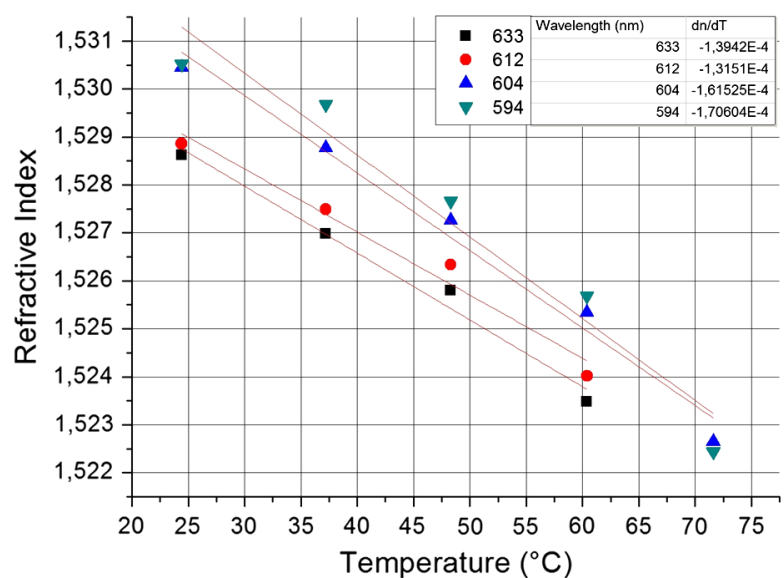

(a)

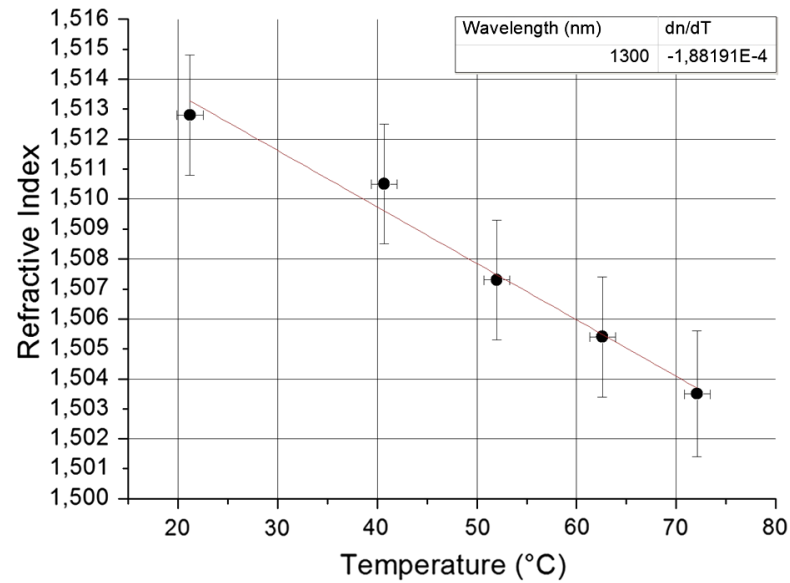

(b)

Fig. 4 (a) Thermal dispersion for the polymer-blend samples obtained by m-lines in the visible spectral region and (b) thermal dispersion for the polymer-blend samples obtained by $\mathrm{m}$-lines at $1300 \mathrm{~nm}$ with error bars.

(independent of the wavelength) between the refractive indices obtained from the two techniques in the order of $5 \times 10^{-3}$. This shift represents a higher measured index by ellipsometry than by m-lines for the guiding material and the inverse case for the buffer material. However, one must take into account the relative uncertainties on measurements from the two methods: in the order of $10^{-2}$ and $10^{-3}$ for ellipsometry and m-lines, respectively, translating to the fact that the absolute index values from m-lines are of a greater precision. The fact that the difference between the refractive indices is constant over all wavelengths considered demonstrates that the dispersion obtained is the same for both ellipsometry and $\mathrm{m}$-lines. A linear correction $\mathrm{A}^{\prime}$ in the Cauchy chromatic dispersion equation is thus applied to the refractive indices from ellipsometry $n_{\text {ellipso }}$ calculated from the m-lines indices $n_{\mathrm{m} \text {-lines }}$ using Eq. (11). This correction factor takes the value of $-7.1 \times 10^{-3}$ for the guiding material and $+4.6 \times 10^{-3}$ for the buffer material. In both cases, therefore, it is lower than the measurement uncertainty of ellipsometry. The resulting corrected dispersion curves are shown in Fig. 3(b).

$n(\lambda)=A+A^{\prime}+\frac{B}{\lambda^{2}}+\frac{C}{\lambda^{4}}$

where $A^{\prime}=\frac{\sum_{i=0}^{m} n_{\mathrm{m}-\text { lines }}\left(\lambda_{i}\right)-n_{\text {ellipsometry }}\left(\lambda_{i}\right)}{m}$,

where $m$ is the number of $\mathrm{m}$-lines measurements.

The dispersion as a function of the temperature has been obtained via the m-lines technique for the guiding material in the visible and near-infrared spectral regions, as shown in Fig. 4. One can see from the obtained slopes of the graphs that the thermo-optic coefficient varies as a function of the considered wavelength. Due to the similar nature of the guiding and buffer materials, as previously discussed, their thermal dispersions are supposed to be identical.

Table 1 regroups the dispersion information for the polymer blend materials. The surface displayed in Fig. 5 has been plotted using the Cauchy coefficients for the guiding material.

As stated previously, the anisotropic properties of the polymer blend films have been measured by m-lines via
Table 1 Cauchy dispersion coefficients for polymer-blend material (wavelengths in nanometer and temperatures in Kelvin).

\begin{tabular}{lcc}
\hline \hline Material & Coefficient & Value \\
\hline Guiding & $a_{1, \text { guiding }}$ & 1.496 \\
& $b_{1, \text { guiding }}$ & 26,100 \\
& $c_{1, \text { guiding }}$ & $-5.86 \times 10^{9}$ \\
Buffer & $a_{1, \text { buffer }}$ & 1.506 \\
& $b_{1, \text { buffer }}$ & 17,224 \\
& $c_{1, \text { buffer }}$ & $-4.5 \times 10^{9}$ \\
Guiding and buffer & $a_{2}$ & $2.40 \times 10^{-4}$ \\
& $a_{3}$ & $-6.15 \times 10^{-6}$ \\
& $b_{2}$ & -885 \\
$b_{3}$ & 12.2 \\
$c_{2}$ & $2.90 \times 10^{8}$ \\
$c_{3}$ & $-3.83 \times 10^{6}$ \\
\hline
\end{tabular}

the combination of measurements in TE and TM polarizations. These films were deposited by sol-gel techniques by spin coating before allowing the solvent to evaporate, and the anisotropy observed is uniaxial in nature, as described in Sec. 3. The birefringence of the film is calculated as follows:

Birefringence $=\Delta n=n_{o}-n_{e}$.

The thickness, ordinary, and extraordinary refractive indices, and the birefringence of thin films composed of the buffer and guide materials are given in Table 2 . 


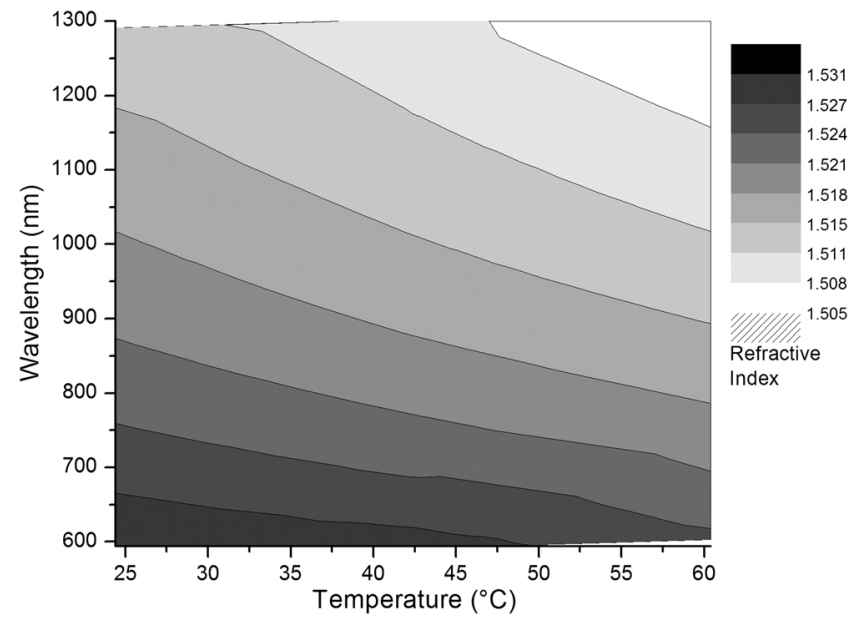

Fig. 5 Dispersion surface for polymer-blend material.

Table 2 Thicknesses, refractive indices, and birefringences for thin films of polymer-blend materials on AF32eco glass substrates.

\begin{tabular}{lcccc}
\hline \hline Film & Thickness $(\mu \mathrm{m})$ & Index $n_{o}$ & Index $n_{e}$ & Birefringence \\
\hline Guide & 4.2 & 1.525 & 1.517 & $8 \times 10^{-3}$ \\
Buffer & 5.5 & 1.521 & 1.508 & $1.3 \times 10^{-2}$ \\
\hline \hline
\end{tabular}

\section{2 $\mathrm{SrTi}_{1-x} \mathrm{Fe}_{x} \mathrm{O}_{3}$ Ceramic Films}

The chromatic dispersion at room temperature $\left(24^{\circ} \mathrm{C}\right)$, as measured by m-lines for the two samples containing $7.5 \%$ and $10 \%$ iron ( $x=0.075$ and 0.1$)$, is shown in Fig. 6(a). The fitted curves represent the dispersion, as given by Cauchy laws. The spectral domain is that covered by the principle emission wavelengths of a $\mathrm{HeNe}$ laser (543 to $633 \mathrm{~nm}$ ). The thermal dispersion and the thermo-optic coefficients measured at $612 \mathrm{~nm}$ for the sample containing $7.5 \%$ iron are given in Fig. 6(b). The limited temperature range is not imposed by the sample but by the m-lines experimental setup. Thermal dilatation, the low melting point of certain components, and the working range of the platinum

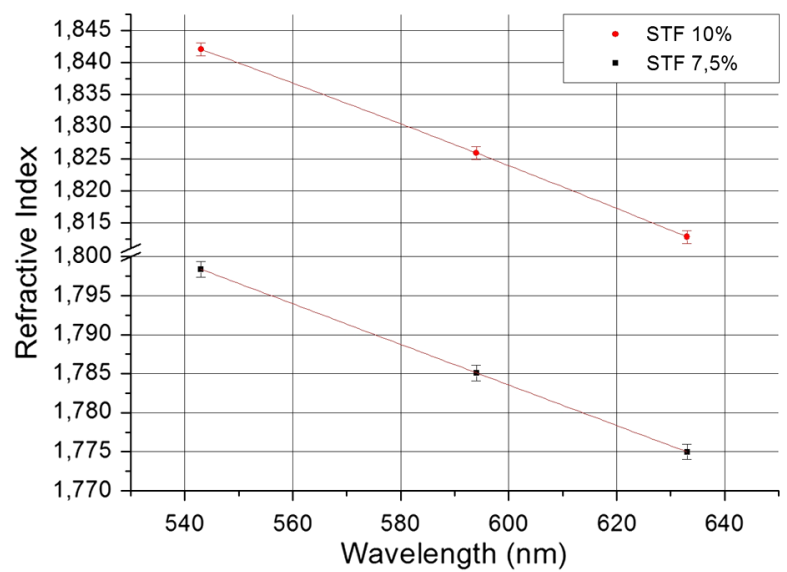

(a) temperature probe rendered it impossible to work at temperatures higher than $170^{\circ} \mathrm{C}$.

The chromatic dispersion measured by ellipsometry at three different temperatures on the sample containing $7.5 \%$ iron is displayed in Fig. 7(a). A zoom on the spectral range covered by the m-lines measurements [Fig. 7(b)] shows the change in refractive index with the very low wavelength in this region. Unfortunately, due to the low resolution of ellipsometry, it was not possible to work in the temperature range as for the m-lines measurements, as the index changes induced were too small to be detected. However, from the refractive index change of approximately $1.5 \times 10^{-2}$ between the ambient temperature and $300^{\circ} \mathrm{C}$, one can estimate a thermo-optic coefficient of $-6 \times 10^{-5} / \mathrm{K}$, roughly half of that obtained from $\mathrm{m}$-lines. It should again be noted that due to the enhanced precision of m-lines measurements, the thermo-optic coefficient obtained by m-lines should be retained. It is also clear that an important change in the material's optical properties occurs between $300 \mathrm{deg}$ and $400 \mathrm{deg}$. This change in behavior will be studied at a later date.

The global photometric parameters (transmittance, reflectance, and absorbance), as measured by integrating sphere, are shown in Fig. 8(a). As previously mentioned, these measurements were used to verify the quality of the ellipsometry results, in particular for the absorption coefficient, which is a parameter that is not easy to measure in thin-film optics. As such, a comparison of the measured photometric coefficients and those calculated from the complex refractive index and the film thickness obtained by ellipsometry are given in Fig. 8(b). A good agreement between the results from the two methods is observed, particularly at wavelengths over $450 \mathrm{~nm}$. Below this wavelength, the ceramic material exhibits strongly varying absorption phenomena. This renders the determination of the refractive index more complicated and requires a more complex dispersion model than that used here.

The imaginary part of the refractive index (extinction coefficient), as calculated by ellipsometry and validated by integrating sphere absorbance measurements, is shown in Fig. 9. One should note that toward higher wavelengths, the extinction coefficient is not zero, which reflects the metallic behavior of the ceramic material caused by the iron

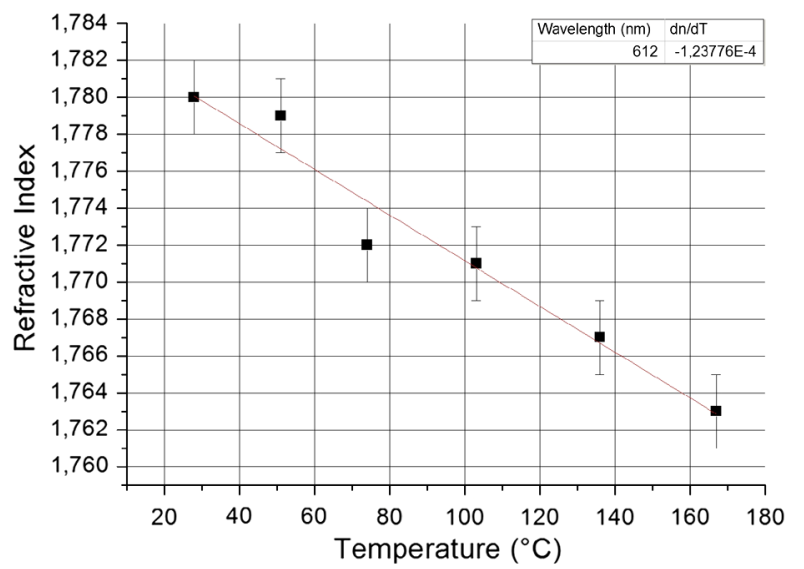

(b)

Fig. 6 (a) Chromatic index dispersion for two doped ceramic samples $(7.5 \%$ and $10 \% \mathrm{Fe})$ at ambient temperature $\left(24^{\circ} \mathrm{C}\right)$ and $(\mathrm{b})$ thermal dispersion for $7.5 \%$ Fe-doped ceramic samples at $612 \mathrm{~nm}$ with error bars. 


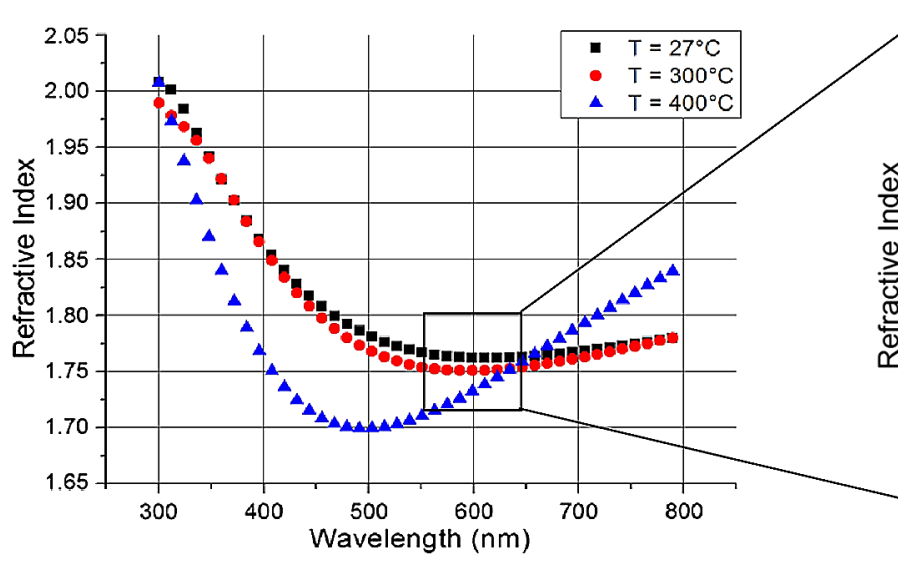

(a)

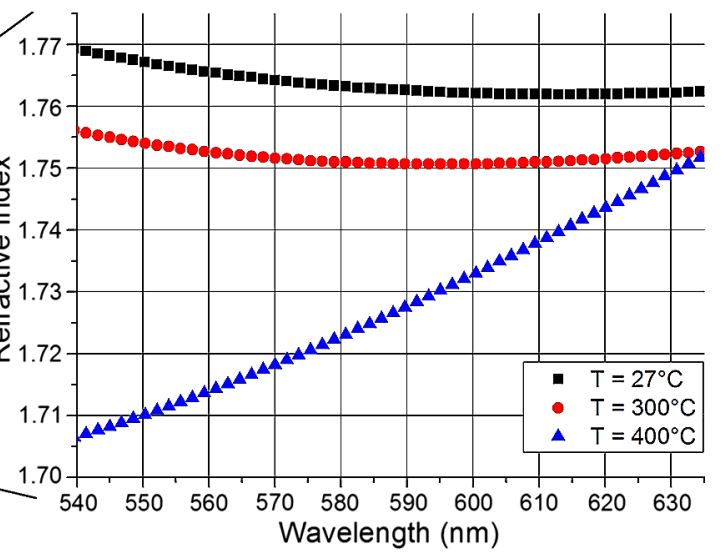

(b)

Fig. 7 (a) Chromatic index dispersion for the ceramic sample doped with 7.5\% Fe at three temperatures and (b) zoom on spectral range covered by m-lines measurements.

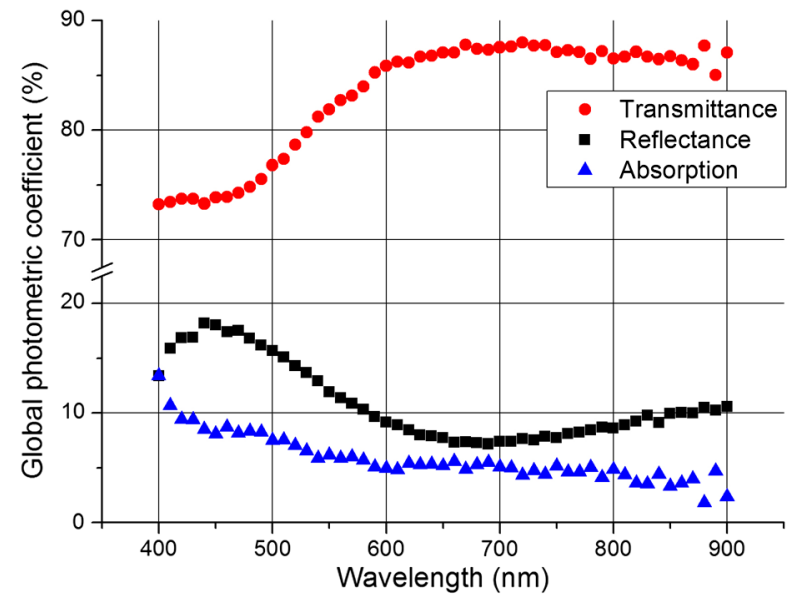

(a)

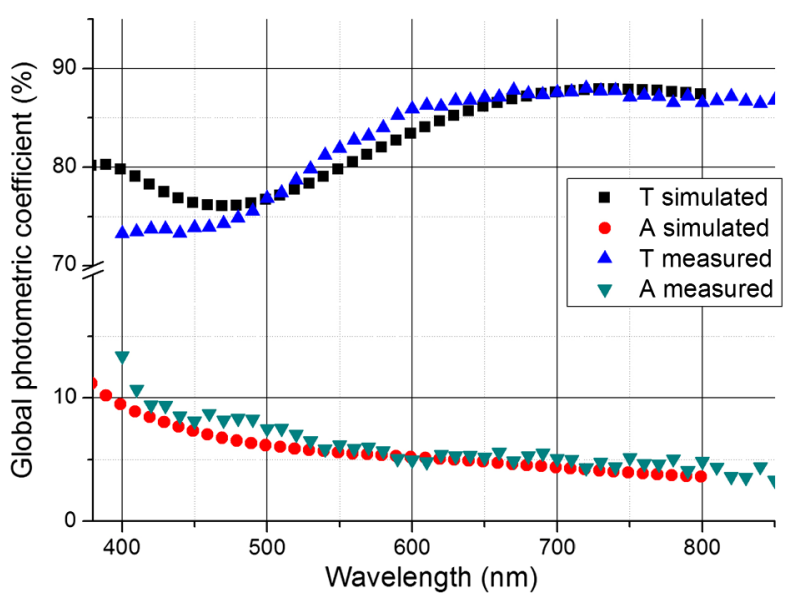

(b)

Fig. 8 (a) Global photometric coefficients (reflectance, transmittance, and absorbance) for the ceramic samples doped with $7.5 \%$ Fe and (b) comparison between measured transmittance and absorbance and simulated values from complex refractive index obtained from ellipsometry.

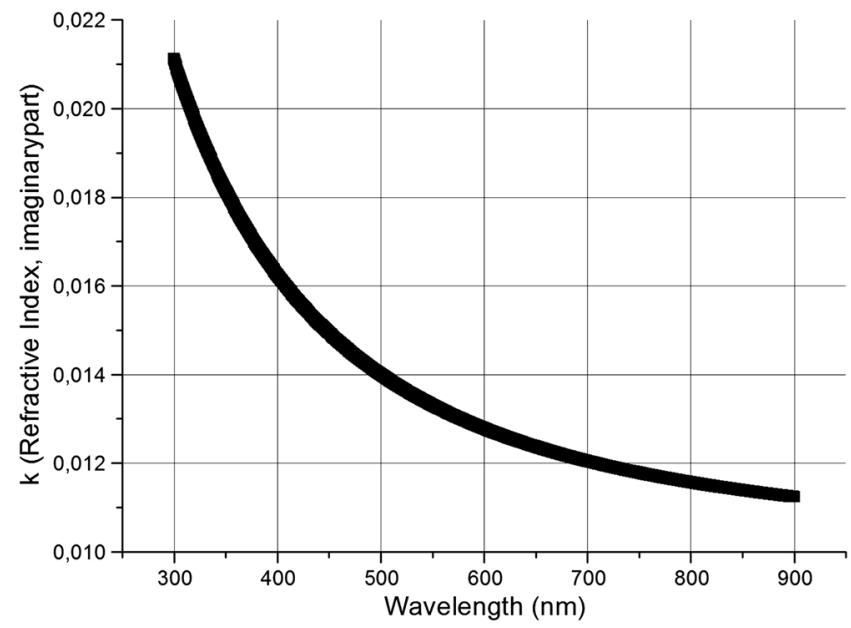

Fig. 9 Extinction coefficient chromatic dispersion for the ceramic sample doped with $7.5 \%$ Fe obtained by ellipsometry. doping. It has been shown that transition metal doping of perovskite-type semiconductors adjusts the Fermi level, such that it is contained within the conduction band. ${ }^{16}$ Therefore, absorption occurs for all photon energies, not just for those with an energy superior to that of the band gap.

Further, m-lines measurements have allowed for the calculation of the Cauchy chromatic and thermal dispersion coefficients, as displayed in Table 3 . The dispersion surface over the employed spectral and temperature ranges is also shown in Fig. 10.

\section{Perspectives}

For thin films deposited by evaporation, such as the ceramic films dealt with in this article, the particles to be deposited that arrive on the substrate tend to form columns of material, in which the angle relative to the substrate plane depends on the orientation of the substrate with respect to the direction of arrival of the incident particle stream. ${ }^{17}$ Given that the void between the columns is an electrical insulator, the resonant frequency of electrons confined in the columns depends on 
Table 3 Cauchy dispersion coefficients for ceramic material doped with $7.5 \% \mathrm{Fe}$ (wavelengths in nanometer and temperatures in Kelvin).

\begin{tabular}{lc}
\hline \hline Coefficient & Value \\
\hline$a_{1}$ & 0.524 \\
$a_{2}$ & 0.0111 \\
$a_{3}$ & $-4.89 \times 10^{-5}$ \\
$b_{1}$ & 955985 \\
$b_{2}$ & -8593.22 \\
$b_{3}$ & 37.229 \\
$c_{1}$ & $-1.814 \times 10^{11}$ \\
$c_{2}$ & $1.647 \times 10^{9}$ \\
$c_{3}$ & $-7.08 \times 10^{6}$ \\
\hline \hline
\end{tabular}

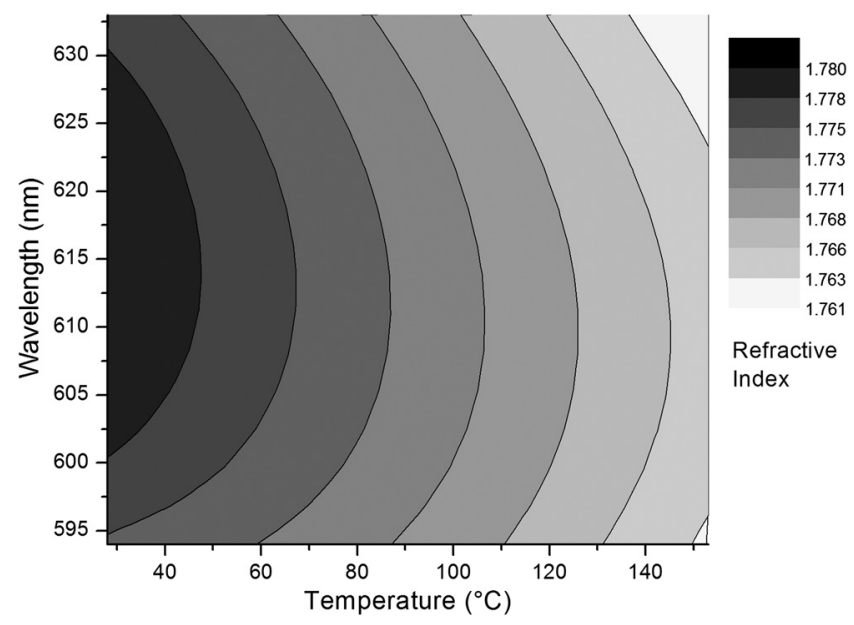

Fig. 10 Dispersion surface for poly ceramic material doped with $7.5 \%$ $\mathrm{Fe}$.

the orientation of the oscillation. As such, electric field components directed following the long axis of the columns experience a different refractive index than those directed following the diameter of the columns. If we consider the simplest case, where the substrate is perpendicular to the arrival direction of particles during the deposition and the columns formed are perpendicular to the substrate plane, the same type of uniaxial anisotropy as observed in constrained solgel films may be observed, as shown in Fig. 11.

We note that if the deposition is carried out for non-normal incidence angles, a biaxial anisotropy may be observed. In this case, one axis follows the long axis of the columns, the second is perpendicular to the long axis of the columns and parallel to the arrival direction of the deposited particles, and the third is orthogonal to the former two axes.

In the future perspectives for this article, we envisage the calculation of the birefringence exhibited by the ceramic semiconductor material deposited by evaporation. This has
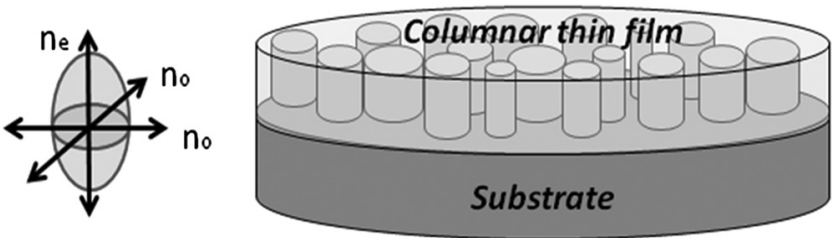

Fig. 11 Uniaxial anisotropy due to the columnar structure of thin films deposited by evaporation techniques.

not been possible for the samples characterized here, as the optical thickness is not sufficient for the films to support more than one guided mode per polarization. The measurement of the birefringence on samples presenting a variety of thicknesses would allow us to infer certain structural properties of the films as a function of their thickness.

\section{Conclusion}

We have shown that the chromatic and thermal dispersions of the refractive index of two different materials can be obtained using a variety of optical methods. The complementary nature of the measurements made has been used, in order to obtain higher than standard degrees of precision, for example the linear correction applied to chromatic dispersion obtained by ellipsometry. The fact that very different materials, a polymer blend and a ceramic semiconductor, have been characterized demonstrates the versatility of the techniques used. Other materials suitable for characterization by these methods include, but are not limited to, metallic oxides, organic polymers, films with nano-structured inclusions, and photoresists.

\section{Acknowledgments}

This work has been possible thanks to funding from the French ANR (Agence Nationale de la Recherche) and the CAPES-COFECUB program, which has facilitated the collaboration with the USP in Brazil (individual program number Ph 699/10). Results presented here for the polymer blend materials have been obtained as part of the ANR PEPS (Pellet Photosensor) project.

\section{References}

1. M. Debliquy, "Capteurs de gaz à semiconducteurs," Tech. l'Ingénieur R2385, 1-16 (2006).

2. T. Mazingue et al., "Nanostructured $\mathrm{ZnO}$ coatings grown by pulsed laser deposition for optical gas sensing of butane," J. Appl. Phys. 98, 074312 (2005).

3. V. R. Mastelaro et al., "Ozone gas sensor based on nanocrystalline $\mathrm{SrTi}_{1-x} \mathrm{Fe}_{x} \mathrm{O}_{3}$ thin films," Sens. Actuators B 181, 919-924 (2013).

4. M. Kakihana, T. Okubo, and M. Arima, "Polymerized complex route to the synthesis of pure $\mathrm{SrTiO}_{3}$ at reduced temperatures: implication for formation of $\mathrm{Sr}-\mathrm{Ti}$ heterometallic citric acid complex," J. Sol-Gel Sci. Technol. 12, 95-109 (1998).

5. P. K. Tien, R. Ulrich, and R. J. Martin, "Modes of propagating light waves in thin deposited semiconductor films," Appl. Phys. Lett. 14, 291-294 (1969).

6. H. Rigneault, F. Flory, and S. Monneret, "Nonlinear totally reflecting prism coupler: thermomechanic effects and intensity-dependent refractive index of thin films," Appl. Opt. 34(21), 4358-4369 (1995).

7. F. Flory, "Guided waves techniques for the characterization of optical coatings," in Thin Films for Optical Systems, F. Flory, Ed., Optical Engineering Series, Vol. 49, pp. 393-454, Marcel Dekker Inc., New York (1995).

8. P. Huguet-Chantôme, L. Escoubas, and F. Flory, "Guided-wave technique for the measurement of dielectric thin-film materials' thermal properties," Appl. Opt. 41(16), 3127-3131 (2002).

9. S. Monneret, P. Huguet-Chantôme, and F. Flory, "m-lines technique: prism coupling measurement and discussion of accuracy for homogeneous waveguides," J. Opt. A: Pure Appl. Opt. 2(3), 188-195 (2000). 
10. I. Tokarev and S. Minko, "Stimuli-responsive hydrogel thin films," Soft Matter 5, 511-524 (2009).

11. H. Tompkins and E. Irene, Handbook of Ellipsometry, William Andrew Publishing, Norwich (2005).

12. R. M. A. Azzam and F. F. Sudradjat, "Reflection coefficients of p- and s-polarized light by a quarter-wave layer: explicit expressions and application to beam splitters," Appl. Opt. 47(8), 1103-1108 (2008).

13. J.-J. Chen, J.-D. Lin, and L.-J. Sheu, "Simultaneous measurement of spectral optical properties and thickness of an absorbing thin film on a substrate," Thin Solid Films 354, 176-186 (1999).

14. K. Ohta and H. Ishida, "Matrix formalism for calculation of the ligh beam intensity in stratified multilayered films, and its use in the analysis of emission spectra," Appl. Opt. 29(13), 1952-1959 (1990).

15. A. N. Bashkatov and E. A. Genina, "Water refractive index in dependence on temperature and wavelength: a simple approximation," Proc. SPIE 5068, 393-395.(2003)

16. X. G. Guo et al., "Electronic band structure of $\mathrm{Nb}$ doped $\mathrm{SrTiO}_{3}$ from first principles calculation," Phys. Lett. A 317, 501-506 (2003).

17. F. Flory and L. Escoubas, "Optical properties of nanostructured thin films," Prog. Quantum Electron. 28, 89-112 (2004).

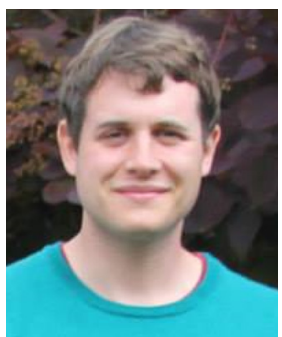

Thomas Wood obtained a masters degree in physics from the University of Manchester (UK) in 2009, having studied abroad for one year at Joseph Fourier University in Grenoble (France). He then completed a $\mathrm{PhD}$ at Aix-Marseille University (France) in 2013, for which the major research topics were the characterization of the chromatic and thermal dispersion of the refractive indices of materials deposited in thin films, and the study of gas sensing systems. Since finishing his $\mathrm{PhD}$, he has been working as a postdoctoral researcher in the IM2NP laboratory, characterizing the optical properties of materials destined to be integrated into organic solar cells. He has presented his work at major international conferences and is the author of 6 accepted journal articles and conference proceedings.

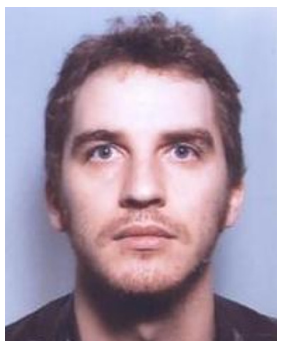

Judikaël Le Rouzo received his $\mathrm{PhD}$ in physics in 2007 from the Ecole Polytechnique, France. His researches are mainly on the optoelectronic devices such as photodetector or photovoltaic solar cells. He is specialized in optics and photonics and performs work on the interaction between light and matter, especially in the cases of nanostructures. More than 15 journal papers and 20 conference papers about his research have been published in the last 5 years.

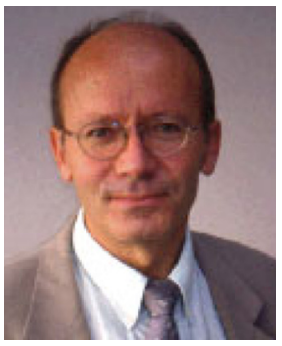

Francois Flory graduated from Ecole Centrale Marseille in 1975 . He received a $\mathrm{PhD}$ in optics in 1978 and a "Thése d'Etat" in 1987. He is now a professor "classe exceptionnelle" at Ecole Centrale Marseille. He was deputy director of this institution for 6 years and co-founded the optical cluster POPsud. $\mathrm{He}$ performs his research at the Institute for Materials, Microelectronics and Nanosciences. His research interests are optical coatings and nanophotonics for different application fields such as solar cells, sensors and light detectors. He is editor and co-author of the book "Thin Films for Optical Systems" (Marcel Dekker, 1995), author of more than 200 publications, book chapters, patents and conferences.

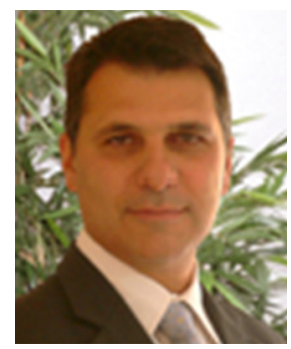

Paul Coudray obtained his $\mathrm{PhD}$ in opto-electronics in November 1993. As the author of more than 70 papers, of which a dozen are invited articles, he created the KLOE company in January 2001. This company develops and produces circuits and integrated optics systems for applications in the domains of sensing, medicine, and biotechnologies, as well as micro-technology direct laser-writing systems.

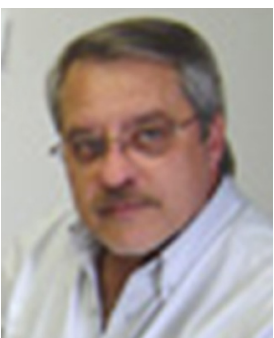

Valmor Mastelaro obtained a bachelors degree in physics from Sao Carlos Federal University in 1985, a masters degree in applied physics from the Instituto de Fisica de Sao Carlos (IFSC) / University of Sao Paulo in 1988, and a doctorate in science from the University Paris XI (Paris Sud) in 1992. He is currently an associate professor at the IFSC, part of the University of Sao Paulo. He has experience in many domains of physics, such as the study of solid crystalline ans amorphous structures, working principally on the following topics: Characterization of local and electronic structure of ceramic materials, nanostructured oxides and vitreous materials, using the following techniques: absorption spectroscopy of x-rays (EXAFSXANES), x-ray diffraction, Raman spectroscopy. He also performs ab-initio calculations aimed at interpreting $x$-ray absorption spectroscopy data.

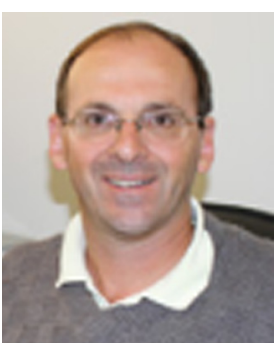

Sérgio C. Zilio research is aimed to the study of linear and nonlinear optical properties of materials and the development of new techniques for that purpose. Ultrafast pulses are employed to investigate the nonlinear refraction and absorption spectrum and dynamics, and to determine photophysical parameters. Coherent control methods are also used in order to enhance optical nonlinerities and optmize ultrafast laser pulses. 